\title{
Molecular beam epitaxy of GaNAs alloys with high As content for potential photoanode applications in hydrogen production
}

\author{
S. V. Novikov, C. R. Staddon, and C. T. Foxon \\ School of Physics and Astronomy, University of Nottingham, Nottingham NG7 2RD, United Kingdom \\ K. M. Yu, R. Broesler, M. Hawkridge, Z. Liliental-Weber, and W. Walukiewicz \\ Materials Sciences Division, Lawrence Berkeley National Laboratory, 1 Cyclotron Road, Berkeley, \\ California 94720-8197 \\ J. Denlinger \\ Advanced Light Source, Lawrence Berkeley National Laboratory, 1 Cyclotron Road, Berkeley, \\ California 94720-8197 \\ I. Demchenko \\ Advanced Light Source, Lawrence Berkeley National Laboratory, 1 Cyclotron Road, Berkeley, \\ California 94720-8197, Department of Chemistry, University of Nevada Las Vegas, 4505 Maryland, \\ Pkwy-Box 454003, Las Vegas, Nevada 89154-4003, and Institute of Physics, PAS, al. Lotnikow 32/46, \\ 02-668 Warsaw, Poland
}

()

The authors have succeeded in growing $\mathrm{GaN}_{1-x} \mathrm{As}_{x}$ alloys over a large composition range $(0<x$ $<0.8)$ by plasma-assisted molecular beam epitaxy. The enhanced incorporation of As was achieved by growing the films with high $\mathrm{As}_{2}$ flux at low (as low as $100^{\circ} \mathrm{C}$ ) growth temperatures, which is much below the normal GaN growth temperature range. Using $\mathrm{x}$-ray and transmission electron microscopy, they found that the GaNAs alloys with high As content $x>0.17$ are amorphous. Optical absorption measurements together with $\mathrm{x}$-ray absorption and emission spectroscopy results reveal a continuous gradual decrease in band gap from $\sim 3.4$ to $<1 \mathrm{eV}$ with increasing As content. The energy gap reaches its minimum of $\sim 0.8 \mathrm{eV}$ at $x \sim 0.8$. The composition dependence of the band gap of the crystalline $\mathrm{GaN}_{1-x} \mathrm{As}_{x}$ alloys follows the prediction of the band anticrossing model (BAC). However, our measured band gap of amorphous $\mathrm{GaN}_{1-x} \mathrm{As}_{x}$ with $0.3<x<0.8$ are larger than that predicted by BAC. The results seem to indicate that for this composition range the amorphous $\mathrm{GaN}_{1-x} \mathrm{As}_{x}$ alloys have short-range ordering that resembles random crystalline $\mathrm{GaN}_{1-x} \mathrm{As}_{x}$ alloys. They have demonstrated the possibility of the growth of amorphous $\mathrm{GaN}_{1-x} \mathrm{As}_{x}$ layers with variable As content on glass substrates.

[]

\section{INTRODUCTION}

The direct conversion of sunlight into hydrogen by photoelectrochemical (PEC) water splitting is one of the direct methods to transfer solar energy into a storable fuel. ${ }^{1}$ PEC cells use photoactive electrodes immersed in an aqueous electrolyte or water. The choice of material for the photoanode is crucial for efficient hydrogen production using the PEC method. The photoanode material needs to be corrosion resistant for prolonged operation. The band gap of semiconductor materials used for photoanodes must be at least 2.0 $\mathrm{eV}$ (Ref. 1) but small enough to absorb most sunlight. In addition to choosing the correct band gap, the conduction and valence band edges must "straddle" the $\mathrm{H}^{+} / \mathrm{H}_{2}$ and $\mathrm{O}_{2} / \mathrm{H}_{2} \mathrm{O}$ redox potentials so that spontaneous water splitting can occur. Gallium nitride $(\mathrm{GaN})$ is a good candidate for this application since it has a band gap of $\sim 3.4 \mathrm{eV}$, high mechanical hardness, and high chemical stability. ${ }^{2}$ Moreover, the band gap of $\mathrm{GaN}$ can be adjusted and decreased due to strong negative bowing in the GaN-based solid solutions with group $\mathrm{V}$ elements. ${ }^{3}$ Therefore, we suggest that the $\mathrm{GaN}_{1-x} \mathrm{As}_{x}$ material system is one of the most promising choices for the photoanodes.

Recently, it has been demonstrated that the electronic structure of the conduction or valence bands of alloys with anions of very different electronegativity can be well described by the band anticrossing (BAC) model. $^{4}$ Due to the substantial difference in the ionization energies of the As and $\mathrm{N}$ anions, the $p$ states of the substitutional As atoms are resonant near the valence band edge of $\mathrm{GaN}$, allowing them to interact strongly with the extended $p$ states of the host. The interaction splits the valence band into a series of $E_{+}$and $E_{-}$subbands with dispersion relations that are dependent on the As concentration. ${ }^{3,4}$ As a result, this effectively reduces the band gap and pushes the valence band edge up toward the $\mathrm{O}_{2} / \mathrm{H}_{2} \mathrm{O}$ potential, while leaving the conduction band above the $\mathrm{H}^{+} / \mathrm{H}_{2}$ potential. On the other hand, in the As-rich GaNAs alloys, the hybridization of the localized $\mathrm{N} s$ states with the GaAs extended $s$ states gives a strong decrease in the conduction band edge. These two effects cause the large bowing across the entire composition range of the $\mathrm{Ga}-\mathrm{N}-\mathrm{As}$ 
alloy system resulting theoretically in an energy gap ranging from $\sim 0.7$ to $3.4 \mathrm{eV}^{3}$ Therefore a GaNAs alloy with a band gap of $\sim 2 \mathrm{eV}$ can be an ideal photoelectrode provided the reduction in the band gap arises primarily from the movement of the valence band and such GaNAs alloys can be grown.

During past 15 years there has been considerable theoretical and experimental interest in As-doped GaN (see review ${ }^{5}$ and references therein). A large miscibility gap was theoretically predicted and experimentally confirmed for the $\mathrm{Ga}-$ $\mathrm{N}$-As system. $\mathrm{GaN}_{1-x} \mathrm{As}_{x}$ alloys at the N-rich end of the phase diagram have been grown by metal-organic vapor phase epitaxy (MOVPE) and by molecular beam epitaxy (MBE) (see references in Ref. 5). For both techniques, it is difficult to obtain a high concentration of As in the alloy before phase separation occurs. The highest concentrations reported in MOVPE layers is $x \sim 0.06$ (Refs. 3 and 6 and in MBE layers is $x \sim 0.01$. $^{7}$

We have recently investigated the growth of $\mathrm{GaN}_{1-x} \mathrm{As}_{x}$ alloys by plasma-assisted molecular beam epitaxy (PA-MBE). ${ }^{8}$ We have succeeded in achieving $\mathrm{GaN}_{1-x} \mathrm{As}_{x}$ alloys over a large composition range by growing the films much below the normal GaN growth temperatures. We found that alloys with a high As content $x>0.1$ are amorphous. Optical absorption measurements reveal a continuous gradual decrease in band gap from $\sim 3.4$ to $\sim 1.4 \mathrm{eV}$ with increasing As content. We have suggested that the amorphous $\mathrm{GaN}_{1-x} \mathrm{As}_{x}$ alloys have short-range ordering that resembles random crystalline $\mathrm{GaN}_{1-x} \mathrm{As}_{x}$ alloys. ${ }^{8}$ These $\mathrm{GaN}_{1-x} \mathrm{As}_{x}$ alloys cover the whole composition range and can be used not only for photoanodes applications in PEC cells for hydrogen production, but also have technological potential for many optical devices operating from the ultraviolet $(\sim 0.4 \mu \mathrm{m})$ to the infrared $(2 \mu \mathrm{m})$, including low cost solar cell applications. The amorphous nature of the GaNAs alloys is particularly advantageous since low cost substrates such as glass can be potentially used for solar cell fabrication.

The aims of the current research were to study the transition from crystalline to amorphous phase in the $\mathrm{GaN}_{1-x} \mathrm{As}_{x}$ alloys, to study the possibility of the growth of amorphous $\mathrm{GaN}_{1-x} \mathrm{As}_{x}$ alloys on low cost glass substrates, and to comprehensively analyze the properties of these new amorphous $\mathrm{GaN}_{1-x} \mathrm{As}_{x}$ alloys.

\section{EXPERIMENT}

GaNAs samples were grown on 2 inch sapphire (0001) substrates by PA-MBE in a MOD-GENII system. The system has an HD-25 Oxford Applied Research rf activated plasma source to provide active nitrogen and elemental $\mathrm{Ga}$ is used as the group III source. In all experiments we have used arsenic in the form of $\mathrm{As}_{2}$ produced by the Veeco arsenic valved cracker.

The MBE system is equipped with reflection high energy electron diffraction (RHEED) for surface reconstruction analysis. For the growth of all GaNAs samples, we have used the same active $\mathrm{N}$ flux [total $\mathrm{N}$ beam equivalent pressure
(BEP) $\sim 1.5 \times 10^{-5}$ Torr] and the same deposition time of 2 h. In order to study the possibility of the growth of amorphous GaNAs alloys on low cost substrates, we have also used standard microscope glass slides $\left(76 \times 26 \times 1 \mathrm{~mm}^{3}\right)$ as a substrate material.

Note that in MBE the substrate temperature is normally measured using an optical pyrometer. However, because we have used uncoated transparent sapphire or transparent glass, the pyrometer measures the temperature of the substrate heater. Therefore in this study we used our estimates for the growth temperature based on the thermocouple readings. ${ }^{8}$

We have used a wide range in situ and ex situ characterization techniques to study the surface morphology, composition, structural, electrical, and optical properties of $\mathrm{GaN}_{1-x} \mathrm{As}_{x}$ layers. The morphology of the samples was studied in situ using RHEED and ex situ using atomic force microscopy (AFM). The structure and orientation of the GaNAs layers was studied by X-ray diffraction (XRD) using the Philips X'Pert Multi Purpose Diffractometer. ${ }^{8}$

Microstructural information on the GaNAs alloys was obtained using transmission electron microscopy (TEM) techniques. Cross-sectional TEM specimens were prepared using standard mechanical grinding and dimpling techniques. A JEOL 3010 with $300 \mathrm{keV}$ accelerating voltage and a resolution of $2.4 \AA$, JEOL CM300 with subangstrom resolution and Philips Tecnai microscope for $Z$-contrast high resolution studies were used. Microscopic crystallinity and phase separation were studied by directly comparing the selective area electron diffraction (SAED) patterns with XRD measurements.

The As content in the GaNAs films was determined by combined Rutherford backscattering spectrometry (RBS) and particle-induced x-ray emission (PIXE) measurements using a $2 \mathrm{MeV}^{4} \mathrm{He}^{+}$beam. Note that the As composition measured by RBS/PIXE is the overall As content in the films but not necessarily As substituting the $\mathrm{N}$ sublattice. The thickness of the films was also measured by RBS and is in the range of 200-1000 $\mathrm{nm}$. The optical properties of the $\mathrm{GaN}_{1-x} \mathrm{As}_{x}$ layers were studied by reflection and absorption measurements in the wavelength range of 200-3000 nm.

The conduction and valence band movements as a function of As incorporation were investigated by the combination of soft x-ray emission (SXE) spectroscopy and x-ray absorption spectroscopy (XAS) that are sensitive to the partial density of states of the valence band and conduction band, respectively. ${ }^{9}$ In this work, the nitrogen $K$ edge (around $409.9 \mathrm{eV}$ ) was investigated at room temperature at the Advanced Light Source on beam line 8.0.1. XAS was detected by the total fluorescence detection mode with an energy resolution of about $0.2 \mathrm{eV}$, and $\mathrm{SXE}$ was measured using the Tennessee/Tulane grating spectrometer with a total energy resolution of $0.6 \mathrm{eV}$. SXE energies were aligned to XAS using elastic emission as a marker for the excitation energy. X-ray intensity $\left(I_{0}\right)$ was measured using a copper grid. XES spectra were normalized to $\mathrm{GaN}$ valence band maximum (VBM) intensity. 

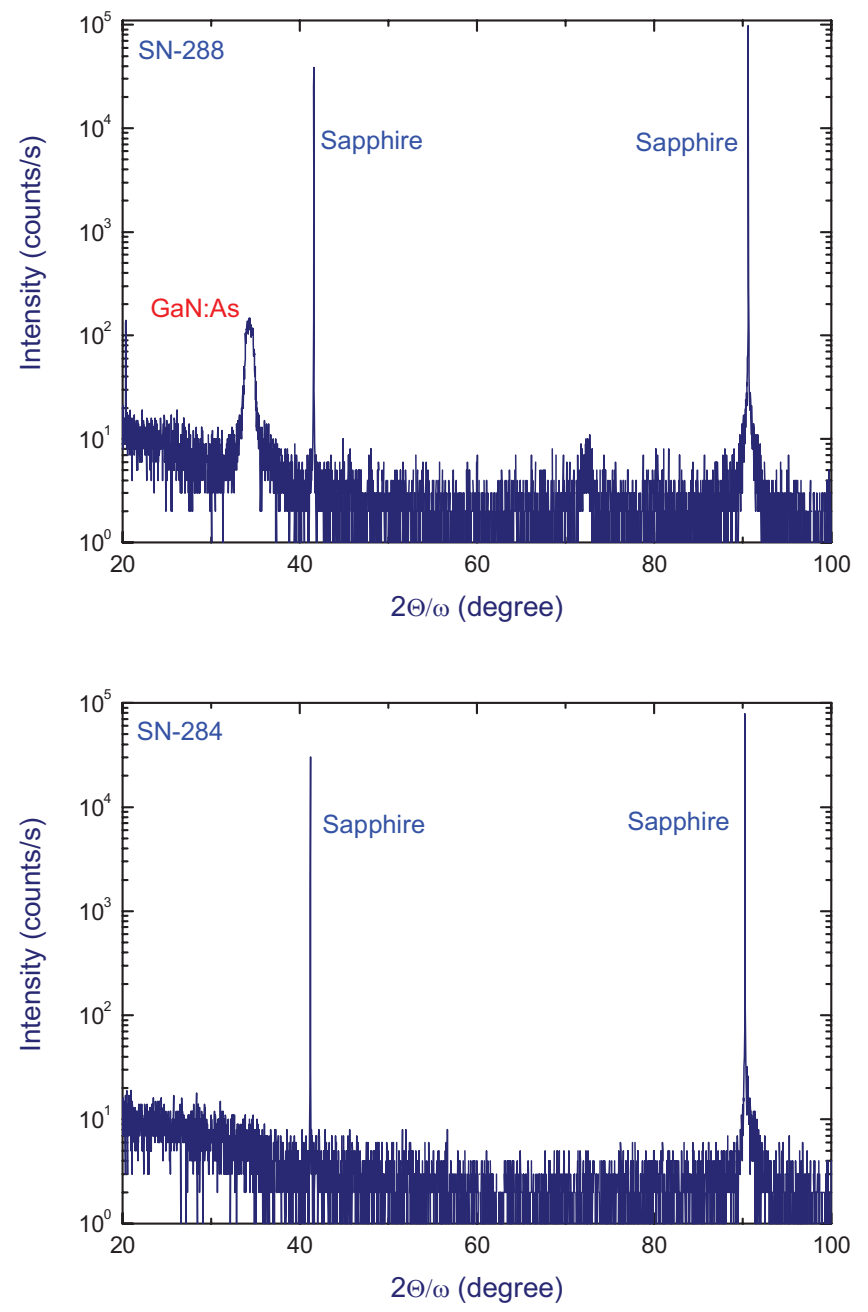

FIG. 1. (Color online) X-ray diffraction data for $\mathrm{GaN}_{1-x} \mathrm{As}_{x}$ layers grown on sapphire substrates at high (a) and low (b) growth temperatures. RBS/PIXE measurements estimate that the As concentrations in $\mathrm{GaN}_{1-x} \mathrm{As}_{x}$ layers are $x \sim 0.08$ (a) and $x \sim 0.45$ (b).

\section{RESULTS AND DISCUSSION}

We have grown a set of GaNAs samples on 2 inch sapphire substrates in a wide range of growth temperatures from $\sim 600$ to $\sim 100{ }^{\circ} \mathrm{C}$. For the growth of GaNAs layers, we used an $\mathrm{As}_{2}$ flux with a BEP $\sim 710^{-6}$ Torr, which is significantly higher than the Ga flux to produce a high As overpressure. All layers were grown under N-rich conditions. The thickness of the GaNAs layers increases with decreasing growth temperature, from $\sim 200$ to $\sim 800 \mathrm{~nm}$. We have single crystal GaNAs layers for the high growth temperatures as confirmed by in situ RHEED during growth and XRD studies after growth. This is evident from the XRD pattern of the GaNAs film grown at $\sim 600{ }^{\circ} \mathrm{C}$ [Fig. 1(a)] showing both the 0002 diffraction peak of $\mathrm{GaN}$ doped with $\mathrm{As}$ at $2 \theta$ $\sim 35^{\circ}$ and peaks from the sapphire substrate.

GaNAs layers grown at low temperatures are amorphous. With decreasing growth temperature the intensity of the 0002 GaNAs diffraction peak decreases and this peak disappears when the layer becomes amorphous, as shown in Fig. 1(b). However, we want to point out that the presence of small

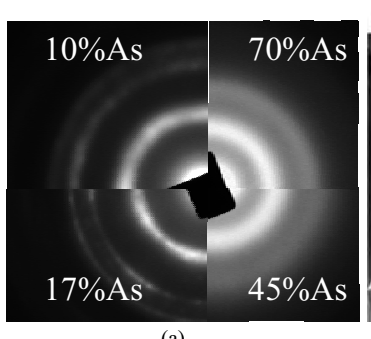

(a)

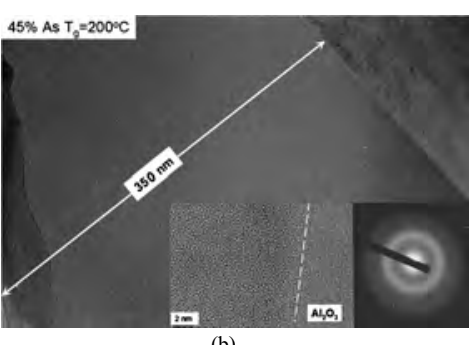

(b)
FIG. 2. SAED pattern from a series of GaNAs samples with increasing As content from $10 \%$ to $70 \%$ showing crystalline to amorphous transition (a). A typical cross-sectional TEM micrograph of an amorphous film $(x \sim 0.45$; $\left.T_{g} \sim 210^{\circ} \mathrm{C}\right)(\mathrm{b})$.

clusters of crystalline regions (a few nanometer in size) that are below the detection limit of XRD cannot be ruled out. AFM studies show that the surface of low temperature grown amorphous GaNAs samples are extremely smooth with a rms roughness as low as $\sim 0.2 \mathrm{~nm}$.

RBS/PIXE measurements estimate that the As concentration in GaNAs layers gradually increases from a few percent in crystalline materials grown at $\sim 500-600{ }^{\circ} \mathrm{C}$ to $>15 \%$ for amorphous films grown at $<400{ }^{\circ} \mathrm{C}$. Thin films with an As content as high as $70 \%$ were grown at the lowest growth temperature of $\sim 100{ }^{\circ} \mathrm{C}$. The As concentrations in the GaNAs layers have been recently confirmed by electron probe microanalyses (EPMAs) studies. EPMA measurements in cross-sectional view also reveal that the composition in the film is uniform in the growth direction within $10 \%$. These results will be published separately.

SAED patterns of GaNAs with increasing As content (decreasing growth temperature) shown in Fig. 2(a) clearly demonstrate that alloys with an As content higher than 17\% are amorphous. Figure 2(b) shows a typical cross-sectional TEM micrograph of an amorphous film $\left(x \sim 0.45 ; T_{g}\right.$ $\sim 210{ }^{\circ} \mathrm{C}$ ) that confirms that the film is uniform and smooth. The high resolution micrograph shown in the inset further confirms the amorphous nature of the film with no observable composition segregation.

Optical absorption and reflection measurements show a progressive shift of the optical band gap to lower energy with decreasing growth temperature. The square of the absorption coefficient $\alpha^{2}$ versus photon energy for $\mathrm{GaN}_{1-x} \mathrm{As}_{x}$ films grown at different temperatures and $\mathrm{As}_{2}$ flux are shown in Fig. 3. The direct band gap of the alloy can be estimated by extrapolating the linear part of the absorption edge down to the energy axis. The overall alloy compositions measured by RBS/PIXE are also given for all layers. Figure 3 shows the samples grown in the temperature range of $100-600{ }^{\circ} \mathrm{C}$ with an $\mathrm{As}_{2}$ flux BEP $\sim 7 \times 10^{-6}$ Torr. Layers with $x$ $<0.17$ are crystalline and with $x>0.17$ are amorphous. Despite the fact that GaNAs layers with $x>0.17$ are amorphous, the energy gap decreases monotonically with increasing As content as the growth temperature decreases.

Soft XAS and soft SXE spectroscopy studies have allowed us to study the position of the conduction and valence bands for $\mathrm{GaN}_{1-x} \mathrm{As}_{x}$ films with the different As contents. 


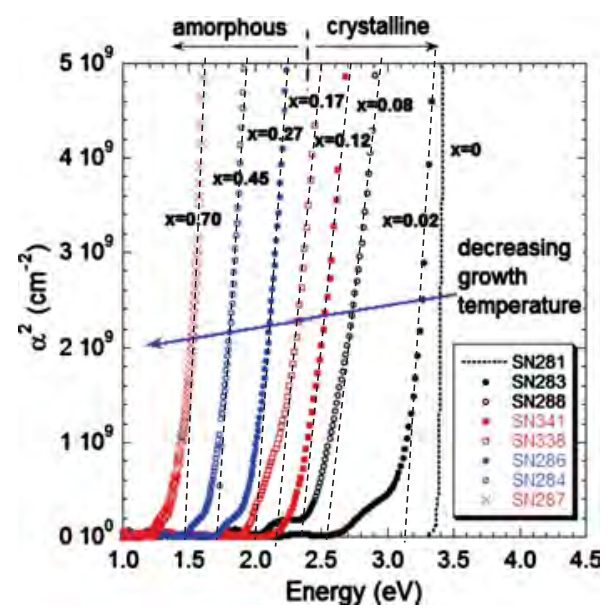

FIG. 3. (Color online) Energy dependence of the square of the absorption coefficient $\left(\alpha^{2}\right)$ for $\mathrm{GaN}_{1-x} \mathrm{As}_{x}$ layers, grown at different temperatures from $\sim 100$ to $\sim 600{ }^{\circ} \mathrm{C}$. The As content in the GaNAs films was determined by combined RBS and PIXE measurements.

The details of these studies will be presented separately. Figure 4 shows a summary for XAS and SXE studies for a set of the GaNAs samples grown at different temperatures. Despite the fact that some of the GaNAs layers are amorphous, we have observed a monotonic decrease in the band gap of $\mathrm{GaN}_{1-x} \mathrm{As}_{x}$ alloys with increasing As concentration. We observed from Fig. 4 that the conduction band moves down and valence band moves up as As composition increases in amorphous GaNAs alloys.

Figure 5 presents the optical band gap of the $\mathrm{GaN}_{1-x} \mathrm{As}_{x}$ films as a function of the overall As content measured by RBS/PIXE. In this figure we have included both crystalline and amorphous films grown under all different growth conditions, both current results and results obtained by us in other sets of GaNAs samples. ${ }^{8}$ Despite the fact that the samples with high As content $x>0.17$ are amorphous, we observe a gradual continuous decrease in band gap from $\sim 3.4$ to $\sim 1.0 \mathrm{eV}$ with increasing As content. The energy gap reaches its minimum of $\sim 0.8 \mathrm{eV}$ at $x \sim 0.8$. Our experimental data from amorphous $\mathrm{GaN}_{1-x} \mathrm{As}_{x}$ follow the dependence predicted by the BAC model rather well. Given the

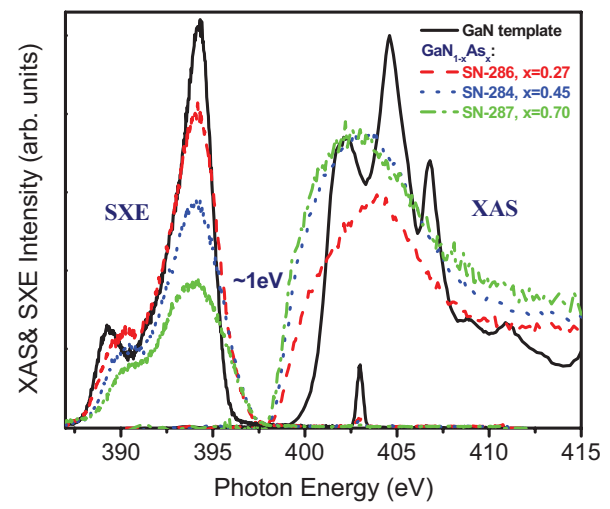

FIG. 4. (Color online) Soft XAS and SXE results for GaNAs samples grown at different temperatures from $\sim 100$ to $\sim 600{ }^{\circ} \mathrm{C}$.

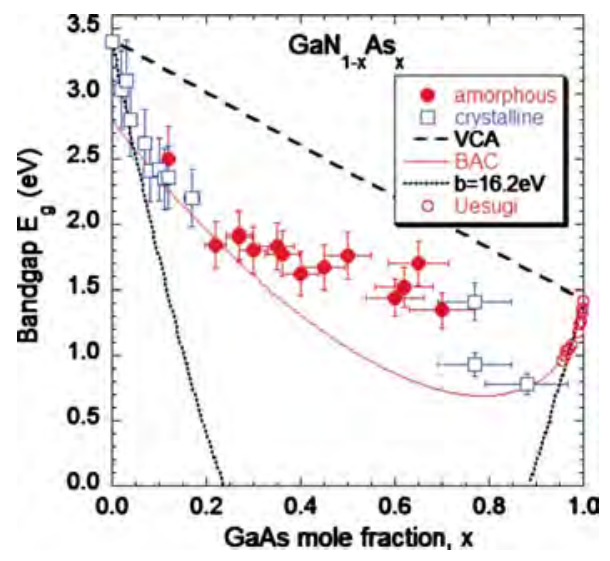

FIG. 5. (Color online) Optical band gap of the $\mathrm{GaN}_{1-x} \mathrm{As}_{x}$ films as a function of the overall As content measured by RBS/PIXE. Calculated composition dependence of the band gap of $\mathrm{GaN}_{1-x} \mathrm{As}_{x}$ alloys based on the BAC model, virtual crystal approximation and using a single bowing parameter extracted from dilute alloys $(b=16.2 \mathrm{eV})$ are also shown. Reported results (Ref. 10) for As-rich GaNAs alloys are also presented.

fact that the calculated band gap in BAC model is an interpolation of results from very dilute crystalline GaNAs and GaAsN alloys, the agreement is remarkable. However, our measured band gap of amorphous $\mathrm{GaN}_{1-x} \mathrm{As}_{x}$ with $0.3<x$ $<0.8$ are larger than that predicted by BAC.

The above results show that amorphous GaNAs alloys may be a good material basis for low cost solar cells. Therefore, we have attempted to grow them on low cost glass substrates. We have used standard microscope glass slides. We have mounted them in the $\frac{1}{4}$ inch Veeco holder designed for $\frac{1}{4}$ of 2 inch wafers. The MBE growth conditions were the same as for the growth on 2 inch sapphire presented above, except we have concentrated only on low growth temperatures to avoid cracking the glass. We were able to grow a set of GaNAs layers at different growth temperatures on these simple glass substrates. In situ RHEED and ex situ XRD measurements on films grown on glass with compositions in the range of $x=0.2-0.55$ confirm that all these film are amorphous. Figure 6 shows a comparison of the absorption data

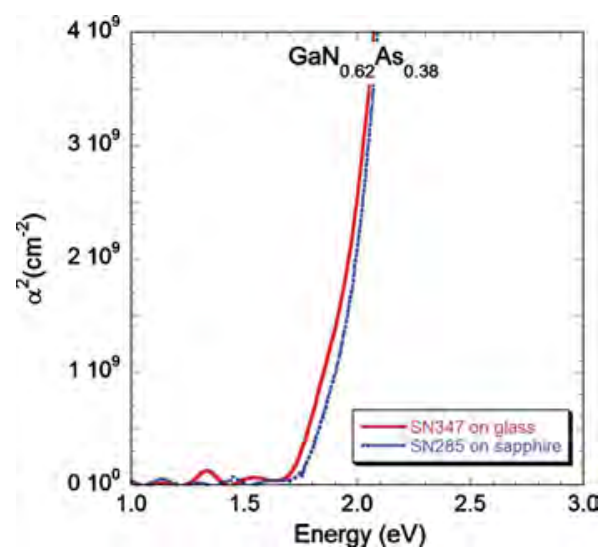

FIG. 6. (Color online) Comparison of the optical absorption data from two $\mathrm{GaN}_{1-x} \mathrm{As}_{x}$ samples with comparable composition $(x \sim 0.38)$ grown on glass and sapphire. 
from two samples with comparable composition $(x \sim 0.38)$ deposited on glass and sapphire. Despite an accuracy of $\sim 10 \%$ in the composition measurements, the optical absorption edges of these two samples are almost identical. This demonstrates the similarity of the electronic structure of the films grown on glass and sapphire substrates.

\section{SUMMARY AND CONCLUSIONS}

We have succeeded in growing $\mathrm{GaN}_{1-x} \mathrm{As}_{x}$ alloys over a large composition range $(0<x<0.8)$ by plasma-assisted MBE. The enhanced incorporation of As was achieved by growing the films with high $\mathrm{As}_{2}$ flux at low (as low as $100{ }^{\circ} \mathrm{C}$ ) growth temperatures, which is much below the normal GaN growth temperature range. Using $x$ ray and TEM we found that the GaNAs alloys with high As content $x$ $>0.17$ are amorphous. Optical absorption measurements together with XAS and SXE results reveal a continuous gradual decrease in band gap from $\sim 3.4$ to $<1.0 \mathrm{eV}$ with increasing As content. The energy gap reaches its minimum of $\sim 0.8 \mathrm{eV}$ at $x \sim 0.8$. The composition dependence of the band gap of the crystalline $\mathrm{GaN}_{1-x} \mathrm{As}_{x}$ alloys follows the prediction of the BAC model. However, our measured band gap of amorphous $\mathrm{GaN}_{1-x} \mathrm{As}_{x}$ with $0.3<x<0.8$ is larger than that predicted by $\mathrm{BAC}$. The results seem to indicate that for this composition range the amorphous $\mathrm{GaN}_{1-x} \mathrm{As}_{x}$ alloys have short-range ordering that resembles random crystalline $\mathrm{GaN}_{1-x} \mathrm{As}_{x}$ alloys. We have demonstrated possibility of the growth of amorphous $\mathrm{GaN}_{1-x} \mathrm{As}_{x}$ layers with variable As content on glass substrates. The large band gap range of the amorphous phase of GaNAs covers much of the solar spectrum and therefore, in addition to the ideal photoelectrode application for N-rich alloys, this material system is a good candidate for full spectrum multijunction solar cells. The amorphous nature of the GaNAs alloys is particularly advantageous since low cost substrates such as glass can be used for solar cell fabrication.

\section{ACKNOWLEDGMENTS}

This work at the University of Nottingham was undertaken with support from the EPSRC (Grant Nos. EP/ G007160/1, EP/G030634/1, EP/G046867/1, and EP/ D051487/1). The work performed at LBNL was supported by the Director, Office of Science, Office of Basic Energy Sciences, Materials Sciences and Engineering Division, of the U.S. Department of Energy under Contract No. DEAC02-05CH11231.

${ }^{1}$ Materials for Energy Conversion Devices, edited by C. C. Sorrell, S. Sugihara, and J. Nowotny (Woodhead, Cambridge, 2005).

${ }^{2}$ Gallium Nitride and Related Semiconductors, edited by J. H. Edgar, S. Strite, I. Akasaki, H. Amano, and C. Wetzel (INSPEC, Stevenage, 1999). ${ }^{3}$ J. Wu, W. Walukiewicz, K. M. Yu, J. D. Denlinger, W. Shan, J. W. Ager, A. Kimura, H. F. Tang, and T. F. Kuech, Phys. Rev. B 70, 115214 (2004). ${ }^{4}$ W. Walukiewicz, K. Alberi, J. Wu, W. Shan, K. M. Yu, and J. W. Ager III, in Physics of Dilute III-V Nitride Semiconductors and Material Systems: Physics and Technology, edited by A. Erol (Springer, Berlin, 2008), Chap. 3.

${ }^{5}$ C. T. Foxon, I. Harrison, S. V. Novikov, A. J. Winser, R. P. Campion, and T. Li, J. Phys.: Condens. Matter 14, 3383 (2002).

${ }^{6}$ A. Kimura, C. A. Paulson, H. F. Tang, and T. F. Kuech, Appl. Phys. Lett. 84, 1489 (2004).

${ }^{7}$ Y. Zhao, F. Deng, S. S. Lau, and C. W. Tu, J. Vac. Sci. Technol. B 16, 1297 (1998).

${ }^{8}$ S. V. Novikov et al., J. Cryst. Growth 311, 3417 (2009).

${ }^{9}$ L. C. Duda, C. B. Stagarescu, J. Downes, K. E. Smith, D. Korakakis, T. D. Moustakas, J. H. Guo, and J. Nordgren, Phys. Rev. B 58, 1928 (1998).

${ }^{10}$ K. Uesugi, N. Marooka, and I. Suemune, Appl. Phys. Lett. 74, 1254 (1999). 


\section{DISCLAIMER}

This document was prepared as an account of work sponsored by the United States Government. While this document is believed to contain correct information, neither the United States Government nor any agency thereof, nor the Regents of the University of California, nor any of their employees, makes any warranty, express or implied, or assumes any legal responsibility for the accuracy, completeness, or usefulness of any information, apparatus, product, or process disclosed, or represents that its use would not infringe privately owned rights. Reference herein to any specific commercial product, process, or service by its trade name, trademark, manufacturer, or otherwise, does not necessarily constitute or imply its endorsement, recommendation, or favoring by the United States Government or any agency thereof, or the Regents of the University of California. The views and opinions of authors expressed herein do not necessarily state or reflect those of the United States Government or any agency thereof or the Regents of the University of California. 